\title{
El sistema de las necesidades y la sociedad civil
}

\author{
(1) Julio de Zan \\ CONICET, Argentina
}

\begin{abstract}
Resumen
El modelo jurídico de la constitución de la sociedad y del estado mediante la ficción de un contrato social es reemplazado por Hegel mediante dos puntos de vista diferentes y complementarios: a) el modelo económico del sistema de las necesidades, que se rige por su propia ley inmanente a la cosa misma de esta esfera, y llega a producir en la sociedad civil una integración real más sólida y a la vez más dinámica que la de las comunidades tradicionales premodernas. b) la integración política sustantiva del espíritu objetivo que hace posible la regulación de los desequilibrios e injusticia de la sociedad civil por el Estado mediante una política moderadamente intervencionista. Más allá del sistema de la competitividad, la sociedad civil es también el continente y la trama de la solidaridad y la beneficencia, la cooperatividad corporativa de artes y oficios, las instituciones públicas no estatales de contralor y de administración de justicia, las actividades públicas del saber y la cultura, la formación de la opinión pública y la actividad de la clase universal que, más allá de la clase política, debe ser independiente del Estado y tiene como fin lo universal en cuanto tal, la formación de las otras clases, especialmente de la clase política y de los funcionarios, el juicio crítico y la contribución a la organización constitucional del Estado.
\end{abstract}

\footnotetext{
Abstract

The juridical model which appeals to the fiction of a social contract for the constitution of society and state is replaced in Hegel's philosophy by two different and complementary points of view: a) the economic model of the system of needs, which is ruled by its own law, immanent to the very thing of this sphere, and which will produce a real integration in civil society that is at the same time more solid and dynamic than the one achieved by traditional pre-modern communities; b) the substantive political integration of the objective spirit, which enables to regulate the imbalances and injustices of civil society through a moderately interventionist policy of the State. Civil society is not reduced to the system of competitiveness, for it is also the container and weave of solidarity, charity, the corporative cooperation of arts and trades, non-state public institutions for control and administration of justice, public activities related to knowledge and culture, the formation of public opinion, and the activity of the universal class, which, with the exception of the political class, must be independent
}

Keywords state economy civil society 
1. Sobre el concepto y las dimensiones de la libertad en Hegel cfr. De Zan (2009: 367-392). Ahora también: De Zan (2015: 93-107). from the State and which final end is the universal itself, the formation of the other classes -in particular the political and civil servants' class-, the critical judgment and the contribution to the constitutional organization of the State.

Hegel ha sido por cierto uno de los grandes apologistas del Estado moderno, pero su filosofía política no es sin embargo estatista sino que está estructurada a partir de la diferencia y la autonomía de la sociedad civil frente al Estado. Este es un rasgo liberal porque la defensa de la sociedad civil frente al estatismo es la nota definitoria del liberalismo. Pero la dinámica de lo político en la época moderna se comprende en Hegel en gran parte como el conflicto y la dialéctica de los términos sociedad civil-Estado, y este será un punto del marxismo. Hegel hace cobrar a la categoría de lo social un estatuto y relieve teórico independiente, como ya lo tenía prácticamente en la realidad histórica de su tiempo y contribuye de esta manera a la constitución de las ciencias sociales (como la economía política y la sociología, además de las ciencias jurídicas y del Estado) que comenzaban a formarse en su época.

El concepto fundamental que expone al comienzo, en la "Introducción" a su Filosofía del Derecho y que atraviesa toda la obra, es el concepto de libertad. Hegel muestra la gran complejidad de "las dimensiones de la libertad" que se han desplegado a través de la historia hasta alcanzar en la sociedad civil y en el Estado moderno la forma reflexiva de una libertad positiva que lleva en su interior el principio de la negatividad y mantiene la independencia frente a las propias decisiones constitutivas de su particularidad. ${ }^{1}$ Esta forma de libertad, que no había sido reconocida por la filosofía de los antiguos puede, considerarse en términos de Hegel como "verdaderamente infinita" (Rph. \22 y 74) Pero aquí quiero poner de relieve el lado de la finitud y las necesidades del hombre (die Bedürfnisse), o las "menesterosidades", como traduce graciosamente don Ramón Valls Plana en su versión española de la Enciclopedia. Esta palabra del castellano antiguo es la mejor traducción de Bedürfnisse, pero como no es un término de nuestro lenguaje usual, y cuesta un poco pronunciarlo, continuaré usando "necesidades".

\section{El funcionamiento sistémico de la economía moderna}

Hegel había aprendido en sus tempranas lecturas de la economía política de Stewart, de Adam Smith y posteriormente de Ricardo, que las necesidades, el trabajo y el intercambio de los productos para la satisfacción de las mismas han formado en la sociedad moderna un sistema, el System der Bedürfnisse, el cual se desarrolla y funciona de manera autónoma. No es del Estado ni de la política que cabe esperar la satisfacción de las necesidades, sino del propio trabajo, y de la participación activa en este sistema, que es el que teje la trama básica de la sociedad moderna. Mediante el avance de la revolución industrial y de la moderna economía de mercado el sistema de las necesidades llega a producir una integración real más sólida y a la vez más dinámica que la de las comunidades tradicionales premodernas y que el modelo contractualista.

El trabajo del hombre como actividad de un individuo singular para la satisfacción de sus propias necesidades es, al mismo tiempo, un trabajo universal, abstracto... Los individuos singulares se limitan a trabajar para una sola de sus necesidades, e intercambian sus productos con los que requieren para la satisfacción de [todas] sus otras necesidades. Su trabajo no tiene por objeto su propia necesidad, sino la abstracción de una necesidad como algo universal; y la satisfacción de la totalidad de sus [otras] necesidades es [el resultado del] trabajo de todos... La satisfacción de las necesidades forma una dependencia universal recíproca de todos con respecto a los demás (HGW, 6). ${ }^{2}$ 
El proceso de socialización se realiza primariamente de ese modo, por la integración de los individuos en este sistema mediante sus capacidades laborales, profesionales, intelectuales, emprendedoras, de gestión empresarial, etc. El modelo jurídico de la constitución de la sociedad y del estado mediante la figura de un contrato, que proviene de Hobbes, es reemplazado por un modelo que podríamos llamar económico. El System der Bedürfnisse comprende lo que hoy llamaríamos: la economía real de la producción, los mercados de bienes y servicios, el mercado de trabajo, y sus relaciones sistémicas con el valor de la moneda y con el sistema financiero; este complejo sistema no puede ser contenido incluso dentro de las fronteras del Estado Nacional, sino que lo abre y lo empuja al exterior a través del mercado internacional, de los flujos migratorios, y la colonización de las más alejadas regiones de la Tierra (Rph. \324).

Jürgen Habermas ha explicitado de manera reflexiva la necesidad de lo que ya Hegel había realizado: emplear dos modelos de análisis diferentes para la compleja realidad de la sociedad y la economía moderna, porque el principio de la libertad, la teoría de la acción y el concepto del espíritu "como un yo que es un nosotros, o un nosotros que es un yo" no son compatibles con el funcionamiento sistémico de la economía de mercado. Entonces tuvo que recurrir a la descripción sistémica del mercado de los economistas escoseses. En Habermas encontramos la misma ruptura entre la teoría de la acción comunicativa y un modelo de análisis diferente para los sistemas en el que los elementos interactúan a través de un determinado medio que es propio y definitorio del sistema, así el dinero es el medio objetivo por el que se producen las operaciones económicas de los mercados como el comercial o el financiero. Hegel había tomado de Adam Smith el modelo de análisis del "sistema de las menesterosidades". Habermas lo toma de la teoría de sistemas de Niklas Luhmann. La base del sistema en la sociología de Luhman es el medio que conecta o comunica a sus elementos, entendiendo "comunicación" como un proceso objetivo no lingüístico.

El ex-alumno de Habermas Axel Honneth en cambio, en su libro El derecho de la libertad. Esbozo de una eticidad democrática (Honneth, 2014), presenta un esquema de análisis único y homogéneo que pretende "actualizar la sistematización hegeliana" (2014: 9-10). La parte central del libro sobre "Las instituciones de la libertad" consta de tres capítulos que se corresponden con las divisiones de la "Eticidad" en la Filosofía del derecho de Hegel. El primero que en Hegel estaba dedicado a "La familia" se titula ahora en Honneth: "El nosotros de las relaciones personales". El segundo, que era el lugar de la sociedad civil hegeliana, se denomina: "El nosotros de la acción de la economía de mercado", con lo cual retrocede el autor al reduccionismo del punto de vista marxista que empobrece la riqueza de la sociedad civil hegeliana. Ya para Hegel además la economía moderna tenía un funcionamiento sistémico y no es adecuado por lo tanto hablar de un "nosotros" como sujeto de la dinámica de los mercados.

El sistema de la economía moderna forma una totalidad independiente del Estado y de lo político, y tiene un funcionamiento sistémico tan complejo que, según Hegel, nadie lo puede abarcar con su mirada, ni los propios actores comunes del sistema, ni el Estado. La incidencia en el sistema de factores contingentes, sociales, técnicos, de catástrofes naturales, o de simples cambios subjetivos en los deseos de los consumidores, o de la moda, hace imprevisibles sus movimientos y tendencias. Su principio son los intereses, y la pasión por los negocios, que Hegel describe como la furia de una bestia salvaje, que no obedece órdenes, a la cual no se la puede conducir mediante decretos de la voluntad del soberano porque sus reacciones instintivas la llevan a escaparse siempre por algún otro lado, y si el Estado se empeña en encerrarla o maniatarla mediante el sistema de la ley puede paralizarla o matarla, anulando así la fuente de su propia subsistencia y del poder del Estado. Es posible sin embargo domesticar la bestia salvaje mediante la habilidad de un domador que la deja correr en libertad conforme a sus instintos, pero encausa y ordena de manera suave y progresiva su carrera desbocada. ${ }^{3}$
3. "La necesidad y el trabajo, elevados a este nivel de universalidad [por la división técnica, social e internacional del trabajo], forman de esta manera por sí mismos en el seno de un pueblo grande un gigantesco sistema de comunidad y dependencia recíproca; pero esta es una vida de lo muerto [es decir, de lo material], que se mueve en sí mismo y que, en su movimiento, se vuelve de manera ciega y elemental de un lado para otro $y$, como una bestia salvaje, necesita de un permanente y severo dominio y domesticación" (HGW 6, p. 234). La bestia salvaje es el mercado, y el domador que tiene que domesticarla y amigarla con la satisfacción de las necesidades y el bienestar de todos es el político. Con esta expresiva metáfora pone de relieve Hegel, al mismo tiempo, la enorme potencialidad del mercado, de su funcionamiento sistémico como el de un organismo vivo, y su peligrosa irracionalidad. Cfr. De Zan (2009: 250 y ss.). 
4. Se cita en adelante con la sigla STP y el número de página. La evolución de las ideas del último Foucault en sus Cursos del Collège de France de los años 1975 a 1979 presentan una proximidad a la filosofía de la economía de Hegel, que es tanto más sorprendente por cuanto Foucault no cita, y quizás no haya leído los textos de Hegel que aquí trabajamos. Para este tema se puede consultar De Zan (2013: Cap. VII, "Los discursos del poder en M. Foucault"), cfr. De Zan (2013: 263-311)
Con otro lenguaje, el último Foucault se acerca a esta concepción cuando habla del gobierno de la sociedad moderna como un arte, y sostiene que "el arte de gobernar es el arte de ejercer el poder en la forma y según el modelo de la economía". La palabra 'economía' designaba incluso en el siglo XVI una forma de gobierno, y recién en el siglo XVIII comenzó a designar un nivel de la realidad social, un campo de intervención del gobierno (Foucault, 2004: 121). ${ }^{4}$ La palabra que Foucault ha puesto en boga para indicar el gobierno, o autogobierno conforme a la racionalidad inmanente de la cosa misma de la economía es: "gubernamentalidad". El Estado que se ha gubernamentalizado ha aprendido que ya no es soberano frente a la economía, que solamente la puede gobernar desde la inmanencia de su propia dinámica, o de su propia ley. "Creo que este es uno de los resultados más importantes de la historia de la razón gubernamental” (STP 327) concluye Foucault después de haber rastreado esta historia a lo largo de su curso del año 1977/78 en el Colegio de Francia. "El poder se ejerce como un arte, o una técnica, y el antiguo soberano se convierte en ingeniero social", o en arquitecto, para evocar la expresión de Aristóteles sobre la política como "ciencia arquitectónica".

La complejidad del sistema de la economía moderna se acrecienta por la división del trabajo y la integración de los mercados a nivel global.

La conexión de este tipo de trabajo fragmentario y aislado con la masa infinita de todas las necesidades [o demandas de consumo de la sociedad] es enteramente inabarcable para la mirada del trabajador, y entraña una dependencia ciega, de tal manera que a menudo una operación en algún lugar lejano, produce de pronto el estancamiento (de la circulación de una mercancía), que hace superfluo e inservible el trabajo de toda una clase de hombres que satisfacía con ese trabajo sus necesidades (HGW 6, 233).

Anthony Giddens describe doscientos años más tarde en términos muy semejantes las consecuencias de la hoy llamada globalización:

Quien estudie las ciudades actuales en cualquier lugar del mundo, sabe que lo que ocurre en un barrio local seguramente ha sido influido por otros factores -como pueden ser los cambios tecnológicos o económicos, y de los mercados de productos- que se han producido en algún otro desconocido lugar del planeta, a una distancia indefinida del barrio en cuestión. La creciente prosperidad del área urbana de Singapur podría estar causalmente relacionada, a través de una complicada red de conexiones económicas mundiales, al empobrecimiento de un barrio de Pittsburg, cuyos productos locales ya no son competitivos en los mercados globales (Guiddens, 1994: 68).

La hermenéutica marxista ha criticado fuertemente la falta de propuestas de Hegel para resolver en su propio ámbito las contradicciones de la economía capitalista, tan agudamente señaladas por él mismo, como la creciente desigualdad, el incremento de la pobreza y la exclusión social, la tendencia a la concentración de capitales, las crisis cíclicas, etc. El señala que la economía necesita una regulación exterior al propio sistema del mercado, mediante políticas impositivas por ejemplo, o créditos de fomento para las pequeñas empresas, etc. Sin la intervención política del Estado, las contradicciones de la economía capitalista, especialmente la creciente desigualdad, la pobreza y la exclusión social llevarán a la autodestrucción de esta sociedad mediante "la rebelión interna y el odio (innere Empörung und Haß)" (HGW 8. 244; HFR 198)5. Pero la necesaria intervención política se limita a proteger a los sectores más débiles de la economía y a paliar las consecuencias negativas del crecimiento, en lugar eliminar las causas de las mismas, y reglamentar o dirigir su funcionamiento. Según las críticas mencionadas, al dejar intactas las estructuras de la economía capitalista, la
5. La sigla HFR remite a la traducción de José María Ripalda del texto HGW 8, Hegel, Filosofía Real, 1984. 
solución política de los conflictos se torna muy poco realista, o pragmáticamente ilusoria.

Es verdad que no encontramos en Hegel un proyecto de reforma estructural de la sociedad existente, pero la historia posterior del S XX parece haberle dado sin embargo la razón porque, al menos por ahora, ha desaparecido del horizonte la perspectiva de un cambio revolucionario de las estructuras socio-económicas, y después del fracaso del socialismo real se ha abandonado en general el proyecto de la creación política de un sistema económico no capitalista. En todo caso la tarea de la Filosofía no es para Hegel la construcción de un modelo ideal de sociedad tal como debe ser. Este concepto de la Filosofía, y la tesis del "Prólogo" de la Filosofía del Derecho: "Lo que es racional es también real, y lo que es real es racional" (Was vernünftig ist, das ist wirklich, und was wirklich ist, das ist vernünftig ${ }^{6}$ ), no le impiden a Hegel sin embargo denunciar con toda fuerza más adelante, en el capítulo del mismo libro sobre la sociedad civil, la irracionalidad y la injusticia de la sociedad existente. La sociedad civil desarrollada (die ausgebildete bürgerlische Gesellschaft) a la que pertenece el filósofo no ha encontrado aún la solución para los graves problemas sociales que son generados por el propio crecimiento de la economía, por lo tanto el reclamo de los pobres es un derecho, una cuestión de justicia. El filósofo puede contribuir mediante la crítica a la apertura del horizonte para el progreso de la historia hacia una sociedad más racional y más justa, y eso es lo que hace Hegel, pero no puede profetizar y menos prescribir el tiempo y la forma de este acontecimiento.

"La importante cuestión de cómo remediar la pobreza es una cuestión suprema que mueve y atormenta a las sociedades modernas" (Rph. $\left.\int 244\right)$.Se discute hoy todavía en los ámbitos académicos de los países centrales si el deber de ayudar a los pobres es un deber meramente moral de los ricos, o si los pobres tienen un derecho a exigir (jurídicamente) una indemnización de la sociedad, y si las sociedades del tercer mundo tienen derechos a cobrarse sobre las del primer mundo. Hegel había dado ya una respuesta a esta pregunta. La caída del individuo en la extrema pobreza puede tener las más diversas explicaciones (Rph. S 241), pero la situación de indigencia y la desocupación de amplios sectores sociales es un problema generado por el propio sistema económico y social, en el cual, "el crecimiento de la riqueza está conectado con la más profunda pobreza" (Hegel, 1967: 84). Ahora bien, puesto que "no se trata de una desgracia natural, sino de una situación social, que depende de los hombres", este desequilibrio que produce la dicotomía social: la marginalidad y la exclusión de un amplio sector (la formación de una plebe), "cobra la forma de una injusticia cometida contra esta o aquella clase". La globalización del sistema plantea hoy este mismo problema entre las sociedades más ricas y las más pobres del mundo como una exigencia de estricta justicia.

El funcionamiento sistémico de la economía como "la totalidad inconsciente y ciega de las necesidades y los modos de su satisfacción", no implica que este sea un sistema sin sujeto. No hay por cierto para Hegel un sujeto colectivo, un macrosujeto del sistema económico (ni del sistema político) como parece pensar por ejemplo Charles Taylor y otros intérpretes. Pero hay por lo menos dos tipos de sujetos individuales que son los principios de la acción: los trabajadores y los burgueses. A quienes hay que agregar los sujetos políticos: los ciudadanos, que en parte se solapan con los dos anteriores. El sujeto del sistema de las necesidades es el ciudadano, pero como burgués, aclara Hegel, es decir como individuo que atiende a la satisfacción de sus necesidades, y que se mueve por sus propios intereses particulares. Pero el resultado del desarrollo del sistema es un efecto de su propia mecánica.

Para comprender la economía y la sociedad moderna, dice en este mismo sentido Foucault, hay que estudiar el nuevo tipo humano que se produce en este contexto,
6. En las recensiones aparecidas inmediatamente después de la publicación de la obra se consideró que esta fórmula era una declarada justificación de la constitución del Estado prusiano, y esta crítica se ha convertido después en un lugar común. E. Ganz respondió sin embargo ya a estas críticas en 1833 mediante la interpretación de ese enunciado como el postulado de que la razón (como la libertad) está siempre presente en la historia y determina el sentido de la verdadera realidad de las cosas, aunque tarde en manifestarse y en configurar el mundo de la facticidad empírica. Para una primera aproximación al debate sobre este enunciado, cfr. Pöggeler et al. (1977: 106). 
el homo oeconomicus: "Una suerte de átomo irremplazable e irreductible de interés... que no puede superponerse, no puede identificarse, ni reducirse a lo que constituye en el pensamiento jurídico lo esencial del sujeto de derecho, no se integra al conjunto del que forma parte de la misma manera que aquel", o de la manera como se vinculan los ciudadanos al formar la unidad política del Estado. "Esta diferencia, esta irreductibilidad del homo oeconomicus a las categorías precedentes... entraña una modificación importante en cuanto al ejercicio del poder soberano del Estado" (Foucault, 2007: 332). Este era ya el problema de la contraposición entre bourgeois y citoyen en Rousseau, y en Hegel.

Los agentes económicos no tienen la capacidad para controlar, ni para prever a mediano o largo plazo, las tendencias funcionales del sistema, deciden bajo incertidumbre y corren los riesgos del fracaso y la quiebra. La ciencia de la economía política conoce las grandes leyes del sistema, y puede acumular datos y mediciones más o menos precisas, pero como actividad del mero entendimiento, "carece de sabiduría", escribe Hegel (HGW 8, 270-271) y no es capaz de realizar predicciones confiables. Solamente la sabiduría práctica del estadista y de los grandes hombres de negocios puede alcanzar aproximaciones intuitivas, que son también falibles, pero cuando aciertan, las inversiones de riesgo producen esas enormes acumulaciones de ganancia del gran capital en la esfera privada, o de las sociedades que tienen la fortuna de salir de la pobreza y el subdesarrollo.

En los textos de Hegel encontramos diversas indicaciones sobre la intervención necesaria del Estado en la economía que permiten reconstruir una clara concepción de política económica moderadamente intervencionista. Pero los graves problemas sociales de la economía moderna, que él había ya denunciado en alguna medida, están por cierto todavía pendientes.

En la segunda parte de esta exposición quiero referirme todavía al espacio de la sociedad civil, pero no ya en relación con el Estado y la economía, sino como el continente y la trama de otras organizaciones, actividades y movimiento públicos y no públicos.

\section{La sociedad civil}

En la genealogía hegeliana el momento más primitivo de la evolución social está representado por la estructura de la relación amo esclavo y la función del poder o el gobierno del oikos era desempeñada por la dominación despótica de un señor. Pero conforme a la dialéctica de la relación de señorío y servidumbre, una vez que los súbditos realizaron el aprendizaje de la obediencia y la disciplina mediante el trabajo esclavo, la primitiva forma de la dominación tiránica se torna superflua, y se produce la inversión de la relación primitiva mediante la lucha por el reconocimiento y autoconciencia de que el régimen de la dominación depende del trabajo y la voluntad de obediencia de los siervos. La situación del reconocimiento recíproco es el estado de derecho en el cual la ley ya no es el mando arbitrario de un tirano, sino una norma racional e igual para todos, a la que están sometidos tanto el pueblo como el gobierno.

Para llegar a ser libres, para adquirir la capacidad de gobernarse a sí mismos, han tenido que atravesar primero los pueblos por la severa disciplina de la sumisión bajo un señor... Las relaciones de señorío y servidumbre y la tiranía han sido un paso inevitable, y bajo este aspecto en cierto modo justificado en la historia (Enciclopedia § 435, Zusatz).

La sociedad civil moderna en cuanto diferente y contrapuesta al Estado es para Hegel el espacio propio del sistema de las necesidades que se rige, como se ha visto, por su 
propia ley inmanente a la cosa misma de esta esfera, que no es ninguna ley natural, como la gravedad, pero tampoco una ley jurídica de la voluntad del legislador, que se puede derogar y remplazar por otra. Pero más allá del sistema económico del trabajo, la producción y los mercados y de las relaciones contractuales privadas, la sociedad civil comprende en la Filosofía del Derecho dos instituciones de carácter público, que son la administración de la Justicia y el poder de policía, cuya concepción y lugar sistemático merecerían un tratamiento aparte. Me limito aquí a destacar que para Hegel estas instituciones no forman parte de la estructura del Estado, sino que cumplen funciones al servicio de la propia sociedad civil y, conforme con la tradición alemana de la Polizeiwissenschaft de los siglos XVII y XVIII, se ubican en este ámbito, ${ }^{7}$ en el cual hay también otras actividades, funciones o expresiones de carácter público. En la sociedad civil coexisten y están directamente conectados por lo tanto lo público y lo privado y no es correcta la identificación de lo público con lo estatal.

Junto al sistema de la competitividad, la sociedad civil es también el espacio de la solidaridad (de las instituciones de beneficencia que se hacían cargo de la mayor parte de lo que hoy son las políticas de bienestar social), y de la acción cooperativa de las corporaciones o gremios. Las corporaciones son para Hegel, juntamente con la familia, los núcleos esenciales de vida comunitaria que mantienen viva "la raíz ética" de la sociedad y del Estado moderno. Si se tiene en cuenta el debate de la Filosofía anglosajona de los años '80 entre el liberalismo y el comunitarismo, podría decirse que Hegel combina elementos de ambas concepciones. El dinamismo de la economía de mercado está movido por el individualismo competitivo y la racionalidad estratégica del homo oeconomicus, pero la disolución de toda comunidad ética sería el retorno a la lucha de todos contra todos del puro estado de naturaleza. Me interesa precisamente puntualizar aquí que, si bien la sociedad civil ha sido considerada como el espacio del libre desarrollo de los intereses particulares, lo económico es solamente un sector de una realidad mucho más amplia y más compleja. Es el espacio de la acción y la creación cultural, de las organizaciones que sostienen los espacios de encuentro de los creadores con su público y de la comunicación en general, de la educación, del saber, y de la formación de la opinión pública, a la cual Hegel presta una especial atención, sobre todo en sus Lecciones.

El capítulo de la Filosofía del Derecho al que me estoy refiriendo, como otros textos anteriores del autor sobre el tema, comprende el esbozo de una sociología de las clases sociales que no se reduce a las posiciones en el sistema económico ni se confunde con las diferencias estamentales de estatus jurídico-político que incidían en sus más antiguos análisis de la sociedad de su época (como en el llamado Sistema de la eticidad de 1801). Hegel elogia la movilidad social que ya manifiesta la sociedad occidental moderna, en la que el individuo tiene el derecho de elegir su lugar entre estas clases sociales diferenciadas por el tipo de actividad particular de cada una de ellas. En este contexto sistemático redefine el autor ahora la categoría del llamado "estamento universal, cuyo negocio son los intereses generales de la sociedad". Sobre este concepto, que ha cambiado notablemente de los escritos tempranos de Hegel a su pensamiento maduro, ${ }^{8}$ quiero proponer una lectura algo diferente.

El estamento universal tiene que estar "descargado del trabajo directo para la satisfacción de sus necesidades particulares, ya sea mediante su patrimonio privado, o mediante compensaciones a cargo del Estado". La consagración al trabajo por lo universal tiene que garantizar a los miembros de esta clase la satisfacción de sus necesidades e intereses privados (Rph. S205). Estas expresiones dan a entender una relación especial de este estamento con el Estado. No se trata de funcionarios en relación de dependencia, que cobran un sueldo estatal, sino de un tipo de profesionales independientes que, en caso de necesidad (como la carencia de fortuna privada)
7. En este contexto la policía es algo bien diferente a la repartición estatal que conocemos hoy con ese nombre. Sobre el significado de esta tradición en la organización y en las formas de gobierno de la sociedad y del Estado moderno puede véase: Foucault (2004: 355-378).

8. En el System der Sittlichkeit había identificado de algún modo el estamento universal con la nobleza del antiguo régimen, o la aristocracia. Pero esta identificación, que aparecía ya entonces como reaccionaria en comparación con sus críticas al antiguo régimen de sus escritos republicanos de juventud, es enseguida abandonada, por lo menos desde la Filosofía Real de $1804 / 05$, y no puede tener cabida en su concepción posterior de la sociedad civil moderna. 
tiene derecho a reclamar una indemnización por las contribuciones que el Estado ha obtenido de su actividad (vom Staat, der seine Thätigkeit in Anspruch nimmt, schadlos gehalten wird). En este parágrafo no especifica Hegel cuales son estas actividades y quienes son los sujetos del estamento universal, por lo que hay que recurrir a otros textos y contextos.

En algunos parágrafos anteriores de este capítulo sobre la sociedad civil había hablado de la Bildung, o de la cultura teórica (es decir del desarrollo del conocimiento), así como de la "cultura práctica" o la adquisición de las "habilidades universalmente válidas para la distintas profesiones" (Rph. S 197). Es claro que las actividades culturales de las ciencias y las artes, cuyo crecimiento independiente del Estado y de la Iglesia es característica de la moderna sociedad civil, no son las ocupaciones propias de ninguna de las otras clases sociales, ni de "la clase sustancial" de los campesinos, ni de la "clase industrial y comercial", sino que corresponden a la llamada clase universal. En el $\int 239$ se refiere a la competencia de la sociedad civil sobre la educación en general, que tiene que complementar la función primaria de la familia y ampliar los horizontes de la formación de los jóvenes para la vida social y laboral, venciendo incluso la posible resistencia del núcleo familiar. "La educación pública como injerencia de la sociedad en el derecho de los padres" se justifica porque "el reclamo de los sujetos singulares de recibir una adecuada formación para poder participar de manera competente en el sistema de las necesidades es también un interés especial de la sociedad civil" (Krautkrämer, 1979: 218).

Las exposiciones orales de Hegel en sus Lecciones permiten ampliar o corregir la identificación del estamento universal con la alta burocracia, o "la clase política" en escritos anteriores. En sus Lecciones de Filosofía del Derecho de Heidelberg de 1817/18 decía: "Al estamento universal pertenecen también los profesores, que se dedican a las ciencias, en aras del progreso general". Es decir que al hablar de este estamento Hegel se incluye claramente a sí mismo dentro de él, pero no se priva de enfatizar que es en interés y honra del propio Estado "el prestar su apoyo especialmente para el desarrollo de la ciencia" (insbesondere zur Ausbildung der Wissenschaft) y hacer posible "el perfeccionamiento de los individuos del estamento general que se dedican a

9. Hegel, Vorlesungen über Naturrecht und Staatwissenschaft, Heidelberg 1917/18, cfr. Hegel (1983: 135). la ciencia a lo largo de toda su vida".9 (Muy lejos está nuestro filósofo por cierto de reconocer con esto al Estado alguna facultad de intervenir en la ciencia, estableciendo por ejemplo prioridades de investigación mediante una política científica, porque tal cosa es un atentado contra la libertad de pensamiento. (Tal infracción no se cometería sin embargo con una política para "las ciencias que no piensan", como diría Heidegger). Las ciencias en las que Hegel está pensando como funciones importantes del estamento universal no son por cierto las hoy llamadas exactas, sino algunas de las ciencias de su Enciclopedia y especialmente las que son objeto de estas lecciones, es decir, la teoría del derecho natural, o filosofía del derecho y la ciencia o filosofía política. Los funcionarios y los miembros del gobierno tienen que estudiar y recibir una educación superior en las humanidades (Studia humaniora), en el derecho y en las ciencias de la administración que los capacite para pensar la cosa pública y actuar con competencia en la gestión de los asuntos de interés común y del Estado (cfr. Krautkrämer, 1979: 245-249).

La función de la clase universal no se limita sin embargo a la formación intelectual y moral de los políticos, porque "el estamento universal tiene una función esencial con respecto a la propia organización del Estado". Esta función tiene que estar "establecida en la constitución política", porque el fin de esta es "lo universal en cuanto tal, de tal manera que el derecho y la seguridad [jurídica] tengan realidad" (Loc.sit.). Estos enunciados sobre el estamento universal no pueden interpretarse como referidos al funcionariado político-administrativo o judicial, sino a quienes forman a estos funcionarios en la educación univesitaria y mantienen la necesaria independencia para 
controlar mediante el juicio crítico el buen ejercicio de las funciones de gobierno; se tienen que referir también especialmente a los autores de las ciencias que están en la base de la formación de los magistrados y hombres de Estado: la filosofía del derecho, la ciencia o filosofía política, el derecho constitucional y la sabiduría jurisprudencial para garantizar los derechos y la seguridad jurídica.

Esta interpretación se ve confirmada, no sólo por el hecho de que la clase universal está ubicada en la sociedad civil, y por lo tanto no es el funcionariado estatal, sino también por el cuidado especial que Hegel pone en asegurar la independencia de esta clase frente al poder político y al Estado. En sus exposiciones orales sobre esta materia se muestra nuestro filósofo especialmente preocupado por los medios para garantizar la autonomía de esta función social, que llamaríamos hoy la función de los intelectuales, para los cuales reclama "ciertos privilegios, o independencia patrimonial, en lugar del otorgamiento de estos privilegios a la nobleza, como suele hacerse en Alemania". En las Lecciones de Berlín de 1821/22 insiste en que para un miembro de la clase universal el mejor medio para preservar su independencia y poder dedicarse a las cosas de interés general es la posesión de una fortuna privada; y de lo contrario tendrá que ser indemnizado por el Estado. "Está claro cual de esos dos casos es el más ventajoso para el Estado". Hegel no se refiere en este párrafo al Estado como institución sino a los funcionarios: "Si lo que busca el Estado son sirvientes (Diener), es mejor que ellos carezcan de patrimonio, porque entonces podrán llegar a ser enteramente dependientes de él". Pero lo que se requiere en cambio de los miembros de la clase universal es que sean

hombres independientes, tanto del sistema de las actividades empresariales, como independientes también del Estado... para que puedan preocuparse lo más libremente posible del interés general y ser activos para este fin (Hegel, 2005:196). 


\section{Bibliografía}

\section{Ediciones de Hegel citadas}

» Hegel, G.W.F.. Gesammelte Werke, Ed. Rheinisch Westfälischen Akademie der Wissenschaften y Deutsche Forschungsgemeinschaft, Hamburg: F. Meiner, 1968 y ss. Las referencias embutidas en el texto con la sigla: HGW, 6 remite al vol. correspondiente de Gesammelte Werke: G.W.F. Hegel: Grundlinien der Philosophie des Rechts (1921). Las referencias a este libro van embutidas en el texto se hacen con la abreviatura Rphy, en lugar de No de página se consigna el parágrafo, como es habitual.

» Hegel, G.W.F. (1984). Filosofía Real. Traducción de J.M. Ripalda. México, Madrid, Buenos Aires: FCE. Se cita con la sigla HFR.

» Hegel, G.W.F. (1967). System der Sittlichkeit, Felix Meiner: Hamburg.

" Hegel, G.W.F. (1983). Vorlesungen über Naturrecht und Staatwissenschaft, Vorlesung v. Heidelberg 1917/18, (Nachgeschrieben v. P. Wannenmann). F. Meiner: Hamburg.

"Hegel, G.W.F. (2005). Die Philosophie des Rechts. Vorlesung von 1821/22. Frankfurt: Suhrkamp.

\section{Bibliografía citada}

"De Zan, J. (2009). La Filosofía social y política de Hegel (367-392). Buenos Aires: Ediciones del Signo.

» De Zan, J. (2013). La vieja y la nueva política. Buenos Aires: UNSAM/J. Baudino Ediciones.

» De Zan, J. (2015). La libertad y el concepto de lo político en Hegel y en las democracias contemporáneas. En Miguel Giusti (Ed). Las dimensiones de la libertad. Sobre la actualidad de la Filosofía del Derecho de Hegel (93-107). Barcelona: Anthropos/ Lima: Fondo Edit PUC.

» Foucault, M. (2004). Seguridad, Territorio y Población. Bs. As./México: FCE.

» Foucault, M. (2007). El nacimiento de la biopolítica (curso de 1978-1979). Bs. As./ México: FCE.

" Giddens, A. (1994). Consecuencias de la Modernidad. Madrid.

"Honneth, A. (2014). El derecho a la libertad. Esbozo de una eticidad democrática. Buenos Aires: Katz.

» Krautkrämer, U. (1979). Staat und Erziehung. En Epimeleia. Beiträge zur Philosophie 10. Munich: J. Berchmans Verlag.

»Pöggeler, O. (1977). (Hrsg.), Hegel, Alber. 